\title{
Review of: "Contribution of obesity and cardiometabolic risk factors in developing cardiovascular disease: a population-based cohort study"
}

Wei-Ting Lin

Potential competing interests: The author(s) declared that no potential competing interests exist.

The topics is interesting and important for adult CVD development. However, I have several comments for this study. I also recommend that the paper receive English language editing by a native speaker.

Methods:

1. Is there any difference of personal characteristics and outcomes at the baseline between exam 1 and exam 2? Why authors evaluated study population from two exams?

2. The abbreviation should be consistent in this study.

3. Please describe how to define current smokers who smoked occasionally.

4. Please clarify the sentence in Definition of covariates part "Demographics, family history of CVDs, .... and mediators, and mediators and CVDs".

5. In clinical and laboratory measurements, "The SBP, DBP and WC were measured at the baseline and every three years intervals". Did author observe any changes of SBP, DBP, and WC at follow-up? Beside $\mathrm{SBP}, \mathrm{DBP}$ and WC, was there any clinical and laboratory index measured every three years intervals?

6. Why only hypertension, high TC, elevate glucose were considered as an independently mediator or combined mediator, excluding high triglycerides and low HDL-C?

\section{Results}

1. In addition to family history of CVDs, was any other family history of cardiometabolic risk factors controlled?

2. Please modify the wording of mediators in tables, such as hypertension, high FPG, and high TC levels.

3. Why the medication use, including hypertension, diabetes, and hyperlipidemia were not adjusted in each model?

4. Please check the description in the footnote of each table.

5. Did authors consider excluding participants who were severe obese? 


\section{Discussion}

1. Aging is highly correlated to the risk of CVD, obesity, and abdominal obesity. How many elderly people (aged $\geq 65$ years) were included in this study? What is the prevalence of obesity, and abdominal obesity, and incidence of CVD in this population?

2. This is an interesting and important study topic. Which cardiometabolic risk factor was found the most important mediator between adiposity and CVD development?

3. Previous findings were addressed in discussions. What different and similar findings of this study were observed compared to these previous studies?

4. The main findings of this study in conclusions should be more concise. 